\title{
Prevalence of microorganism and emergence of bacterial resistance in ICU of Bangabandhu Sheikh Mujib Medical University of Bangladesh
}

\author{
Montosh Kumar Mondal ${ }^{1 *}$, Beauty Rani Roy ${ }^{2}$, Sabina Yeasmeen ${ }^{1}$, Faizul Haque ${ }^{1}$
}

A.K. Qumrul Huda ${ }^{3}$, Debabrata Banik ${ }^{4}$

${ }^{1,3,4}$ Department of Anaesthesia, Analgesia and Intensive Care Medicine. Bangabandhu sheikh Mujib Medical University.
Dhaka, Bangladesh, ${ }^{2}$ OGSB Hospital, Institute of Child Health and Reproductive centre, Mirpur, Dhaka.

Corresponding author: E mail - montoshmondal@yahoo.com

\begin{abstract}
Background Antibiotic resistant bacterial nosocomial infections are a leading problem in intensive care units (ICU).
\end{abstract}

Objective To study the pattern of microorganism and bacterial resistant to antibiotic in ICU of Bangabandhu sheikh Mujib Medical University of Bangladesh.

Methods This retrospective study was conducted in ICU of Bangabandhu Sheikh Mujib Medical University, Bangladesh from January 2010 to December 2012. Total number of samples were 448. The samples of tracheal aspirate, blood and urine for culture and sensitivity was collected from the patient admitted in ICU. Analysis of tracheal aspirate, blood and urine culture was done from hospital record. All bacteria was identified by standard microbiological methods, and their antibiotic sensitivity was performed using disk diffusion method.

Results Total number of samples 448. Samples of tracheal aspirate was 159, positive culture 121(76\%), most frequent identified organism was acenetobacter $45.45 \%$, followed by pseudomonas $32.23 \%$, proteus $11 \%$, klebsiella $10 \%$ and E.coli $3 \%$, samples of blood culture was 148 , positive culture $22(14.86 \%)$, most frequent identified organism was pseudomonas $63.63 \%$, followed by acenetobacter $22.72 \%$, salmonella $4.54 \%$ and E.coli $4.54 \%$ and samples of urine culture was 141, positive culture $36(25.53 \%)$ most frequent identified organism was enterococcus $22.22 \%$, followed by acenatobacter $19.44 \%$, candida $16.66 \%$, klebsiella $13.88 \%$ and E.coli $13.88 \%$. Drug resistant organism of tracheal aspirate was $12(20.33 \%)$ in 2010, 2(20\%) in 2011 and 13(25\%) in 2012. only collistin sensitive organism identified was 28(23.14\%).

Conclusion From this study we concluded that most common site of infection was respiratory tract and most prevailing organism was acinetobacter \& pseudomonas and antibiotic resistant infection is increasing and at present around one fourth organisms were resistant to all antibiotics.

Keywords Microorganism, drug resistance, respiratory tract, acinetobacter and pseudomonas aeruginosa.

(JBSA 2013; 26(1): 20-26)

\section{Introduction}

Increasingly rapid emergence and dissemination of antimicrobial-resistant bacteria has become a world wide problem during the last decades. The intensive care unit often is called the epicenter of infections, due to its extremely vulnerable population of reduced host defences deregulating the immune responses and increased risk of becoming infected through multiple procedures and use of invasive devices distorting the anatomical integrity-protective barriers of patients likeintubation, mechanical ventilation, vascular access, etc. In addition, several drugs may be administered, which also predispose for infections, such as pneumonia, e.g., by reducing the cough and swallow reflexes (sedatives, muscle relaxants) 
or by distorting the normal nonpathogenic bacterial flora (e.g., stress ulcer prophylaxis) ${ }^{1}$.

Consequently, the ICU population has one of the highest occurrence rates of nosocomial infections (20-30\% of all ICU-admissions $)^{2,3}$, leading to an enormous impact on morbidity, hospital costs, and often, survival ${ }^{4-6}$. Along with the problem of nosocomial infection goes the burden of "multidrug" antimicrobial resistance (MDR). The ongoing emergence of resistance in the community and hospital is considered a major threat for public health. Antibiotic resistance increases the morbidity and mortality associated with infections and contributes substantially to rising costs of care, resulting from prolonged hospital stays and the need for more expensive drugs.

Both infection and MDR result in a considerable clinical and economic burden. As such, the presence of MDR boosts the deleterious impact of nosocomial infection ${ }^{7}$. Compared with infections not caused by MDR microorganisms, the additional cost of multidrug resistance in hospitalized patients with infections has been estimated at $\$ 6,000$ to $\$ 30,000$ (per patient) in Belgium ${ }^{8}$. This burden of resistance, however, is probably more due to the higher rate of inappropriate empiric antimicrobial treatment associated with infections caused by MDR pathogens than with the virulence of particular MDR strains ${ }^{9}$.

We are currently faced with (multi drug) resistant bacteria that are difficult and sometimes impossible to treat ${ }^{10}$. The tremendous therapeutic advantage afforded by antibiotics is being threatened by the emergence of increasingly resistant strains of microbes ${ }^{11}$. The problem has recently been worsened by the steady increase in multi-resistant strains and by the restriction of antibiotic discovery and development programs ${ }^{10}$.

In hospital settings world-wide Intensive care Units are faced with rapid and increasing resistance of bacteria. ICUs are the source and site of development of multidrug resistant (MDR) bacteria.

The widespread use of antibiotics both inside and outside of medicine is playing a significant role in the emergence of resistant bacteria ${ }^{12}$. Antimicrobials have transformed our ability to treat many infectious diseases that were killers only a few decades ago. The increasing use of antimicrobials in humans, animals, and agriculture has resulted in many pathogens developing resistance to these powerful drugs ${ }^{13}$. A number of factors contribute to the emergence of antimicrobial resistance in ICUs, including extended length of hospital stay, and the widespread use of prophylactic and therapeutic anti-infective agents. Bacteria have developed resistance to all different classes of antibiotics discovered to date. The most frequent type of resistance is acquired and transmitted horizontally via the conjugation of a plasmid ${ }^{14}$. In recent times new mechanisms of resistance have resulted in the simultaneous development of resistance to several antibiotic classes creating very dangerous multidrugresistant (MDR) bacterial strains, some also known as "superbugs" 15 . The need for new antimicrobial agents is greater than ever because of the emergence of multidrug resistance in common pathogens, the rapid emergence of new infections, and the potential for use of multidrugresistant agents in bioweapons ${ }^{16}$.

Controlling the spread of resistance requires the collaboration of several participants such as Medical, Veterinary and Public Health Communities ${ }^{17}$. Multidrug resistant organisms (MDROs) are resistant to one or more classes of antimicrobial agents and the knowledge of susceptibility pattern is helpful in selecting the empirical therapy and improving the likelihood of a satisfactory outcome for patient ${ }^{18}$. The objective of this study was to determine bacterial pathogens prevalence and to assess the multi-drug resistant (MDR) strains to different antibiotics in the intensive care unit of Bangabandhu Sheikh Mujib Medical University.

\section{Materials}

This retrospective study was carried out in the intensive care unit of Bangabandhu Sheikh Mujib Medical University, Dhaka, Bangladesh from 20102012. The approval of the University ethical committee was taken before conducting the study. Data of total 448 samples (tracheal aspirate 159, blood culture 148 and urine culture 141) for culture and sensitivity were collected from intensive care unit record. The pattern of microorganism and bacterial resistant to antibiotic was recorded. All bacteria was identified by standard microbiological methods, and their antibiotic sensitivity was performed using disk diffusion method.

Sample Collection: Tracheal aspirate, blood and urine specimens were collected aseptically for bacteriological examination from the patient of Intensive care unit of BSMMU. 


\section{Isolation and Identification}

Tracheal aspirate, blood and urine specimens were cultured into blood agar and MacConkey agar media. Bacteriological smears were prepared from the growing colonies then stained with gram stain for morphological identification. All the bacterial isolates were preserved on nutrient agar slants at $4^{\circ} \mathrm{C}$ and subcultured periodically. The obtained pure cultures were identified biochemically.

\section{Antimicrobial Susceptibility Test}

Antimicrobial susceptibility pattern was performed using disk diffusion method on Muller Hinton agar plate. The isolates were tested against amoxicillin, cotrimoxazole, ceftazidime, Cefotaxime, Cefuroxime, Aztreonam, Netilmicin, Ticarcillin, Cephradine, gentamicin, imipenem, ciprofloxacin, ceftriaxone, amikacin, Colistin Sulphate, Tazobactam+ piperacillin.

The proportion of susceptible organisms was calculated as the sum of susceptible isolates relative to the total number of organisms tested. The organism considered as multidrug resistant if it is resistant to three or more antimicrobials.

\section{Results}

Table I Tracheal aspirate-2010, prevalence \& pattern of organism, $n=74$

\begin{tabular}{llcc}
\hline Positive c/s & $\begin{array}{l}\text { Name of } \\
\text { organism }\end{array}$ & Frequency & Percentage \\
\hline $59(79.7 \%)$ & Acenatobacter & 32 & 54.2 \\
& Pseudomonas & 24 & 40.7 \\
& Proteus & 2 & 3.3 \\
& Klebsiella & 1 & 1.69 \\
\hline
\end{tabular}

Table II Tracheal aspirate-2010, pattern of antibiotic sensitivity, $n=59$

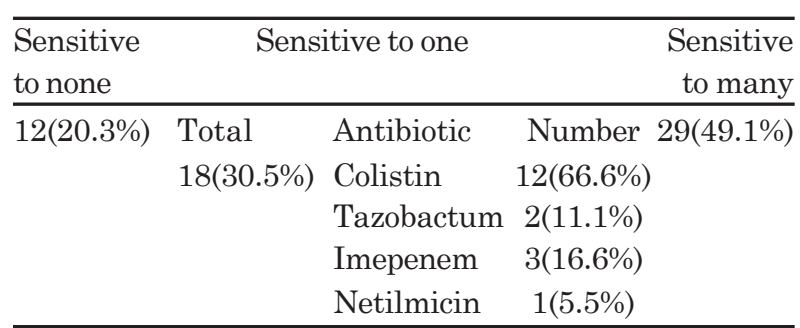

Table III Blood-2010, prevalence \& pattern of organism, $n=61$

\begin{tabular}{llcc}
\hline Positive c/s & Name of organism & Frequency & Percentage \\
\hline $13(21.3 \%)$ & Pseudomonas & 8 & 61.5 \\
& Acenatobacter & 3 & 23 \\
& E coli & 1 & 7.6 \\
& Salmonella & 1 & 7.6 \\
\hline
\end{tabular}

Table IV Blood -2010, pattern of antibiotic sensitivity, $n=13$

\begin{tabular}{|c|c|c|c|c|}
\hline \multicolumn{2}{|c|}{$\begin{array}{l}\text { Sensitive } \\
\text { to none }\end{array}$} & \multicolumn{2}{|c|}{ Sensitive to one } & \multirow{2}{*}{$\begin{array}{c}\text { Sensitive } \\
\text { to many } \\
6(46.15 \%)\end{array}$} \\
\hline \multirow[t]{3}{*}{$1(7.6 \%)$} & Total & Antibiotic & Number & \\
\hline & 6 & Colistin & $3(50 \%)$ & \\
\hline & & Ceftazidim & $3(50 \%)$ & \\
\hline
\end{tabular}

Table V Urine -2010, pattern of antibiotic sensitivity, $n=54$

\begin{tabular}{llcc}
\hline Positive c/s & Name of organism & Frequency & Percentage \\
\hline $19(35.1 \%)$ & Acenatobacter & 6 & 31.5 \\
& Enterococcus & 5 & 26.3 \\
& E coli & 3 & 15.7 \\
& Pseudomonas & 2 & 10.5 \\
& Klebsiella & 2 & 10.5 \\
& Candida & 1 & 5.2 \\
\hline
\end{tabular}

Table-VI Urine -2010. pattern of antibiotic sensitivity, $n=19$

\begin{tabular}{lllcc}
\hline $\begin{array}{l}\text { Sensitive } \\
\text { to none }\end{array}$ & & Sensitive to one & $\begin{array}{r}\text { Sensitive } \\
\text { to many }\end{array}$ \\
\hline $5(26.3 \%)$ & Total & Antibiotic & Number & $9(47.3 \%)$ \\
& $5(26.3 \%)$ & Colistin & $3(60 \%)$ & \\
& & Imipenem & $1(20 \%)$ & \\
& & Ciprofloxacin & $1(20 \%)$ & \\
\hline
\end{tabular}

Table-VII Tracheal aspirate-2011, prevalence \& Pattern of Organism $n=14$

\begin{tabular}{llcc}
\hline Positive c/s & Name of organism & Frequency & Percentage \\
\hline 10(71.4\%) & Acenetobacter & 5 & 50 \\
& Proteus & 2 & 20 \\
& Pseudomonas & 1 & 10 \\
& Klebsiella & 1 & 10 \\
& Streptococcus & 1 & 10 \\
\hline
\end{tabular}

Table VIII Tracheal aspirate-2011, Pattern of antibiotic sensitivity, $n=10$

\begin{tabular}{lllll}
\hline $\begin{array}{l}\text { Sensitive } \\
\text { to none }\end{array}$ & & Sensitive to one & $\begin{array}{r}\text { Sensitive } \\
\text { to many }\end{array}$ \\
\hline $2(20 \%)$ & Total & Antibiotic & Number & $4(40 \%)$ \\
& $4(40 \%)$ & Colistin & $4(100 \%)$ & \\
\hline
\end{tabular}


Table IX Blood-2011, prevalence \& pattern of organism $n=16$

\begin{tabular}{llcc}
\hline Positive c/s & $\begin{array}{l}\text { Name of } \\
\text { organism }\end{array}$ & Frequency & Percentage \\
\hline $3(18.7 \%)$ & Pseudomonas & 2 & 66.6 \\
& Salmonella & 1 & 33.3 \\
\hline
\end{tabular}

Table X Urine-2011, prevalence \& pattern of organism $n=14$

\begin{tabular}{llcc}
\hline Positive c/s & $\begin{array}{l}\text { Name of } \\
\text { organism }\end{array}$ & Frequency & Percentage \\
\hline $4(28.5 \%)$ & Candida & 2 & 50 \\
& Enterococcus & 1 & 25 \\
& Acenetobacter & 1 & 25 \\
\hline
\end{tabular}

Table XI Tracheal aspirate-2012, prevalence \& pattern of organism $n=71$

\begin{tabular}{llcc}
\hline Positive c/s & $\begin{array}{l}\text { Name of } \\
\text { organism }\end{array}$ & Frequency & Percentage \\
\hline $52(73.23 \%)$ & Acenetobacter & 18 & 34.61 \\
& Pseudomonas & 14 & 26.92 \\
& Klebsiella & 8 & 15.38 \\
& Proteus & 7 & 13.46 \\
& Enterococcus & 1 & 1.92 \\
& E. coli & 3 & 5.76 \\
& Gram +ve cocci & 1 & 1.92 \\
\hline
\end{tabular}

Table XII Tracheal aspirate-2012,Pattern of antibiotic sensitivity, $n=52$

\begin{tabular}{|c|c|c|c|c|}
\hline \multicolumn{2}{|l|}{$\begin{array}{l}\text { Sensitive } \\
\text { to none }\end{array}$} & \multicolumn{2}{|c|}{ Sensitive to one } & \multirow{2}{*}{$\begin{array}{l}\text { Sensitive } \\
\text { to many } \\
26(50 \%)\end{array}$} \\
\hline \multirow[t]{3}{*}{$13(25 \%)$} & Total & Antibiotic & Number & \\
\hline & $13(25 \%)$ & Colistin & $12(92.7 \%)$ & \\
\hline & & Tazobactu & n $1(7.7 \%)$ & \\
\hline
\end{tabular}

Table XIII Blood-2012, prevalence \& pattern of organism $n=71$

\begin{tabular}{llcc}
\hline Positive c/s & $\begin{array}{l}\text { Name of } \\
\text { organism }\end{array}$ & Frequency & Percentage \\
\hline $6(8.45 \%)$ & Pseudomonas & 4 & 66.6 \\
& Acinetobacter & 2 & 33.3 \\
\hline
\end{tabular}

Table XIV Urine-2012, prevalence \& pattern of organism $n=73$

\begin{tabular}{llcc}
\hline Positive c/s & $\begin{array}{l}\text { Name of } \\
\text { organism }\end{array}$ & Frequency & Percentage \\
\hline $13(17.80 \%)$ & Pseudomonas & 2 & 15.38 \\
& Enterococcus & 2 & 15.38 \\
& E.coli & 2 & 15.38 \\
& Klebsiella & 3 & 23.07 \\
& Candida & 3 & 23.07 \\
& Proteus & 1 & 7.06 \\
\hline
\end{tabular}

Table XV Prevalence of positive culture of trachea, blood and urine-comparison during 2010-2012

\begin{tabular}{lccccc}
\hline \multicolumn{2}{c}{ Tracheal aspirate } & \multicolumn{2}{c}{ Blood } & \multicolumn{2}{c}{ Urine } \\
Total Sample & Positive c/s & Total Sample & Positive c/s & Total Sample & Positive c/s \\
\hline 159 & $121(80.66 \%)$ & 148 & $22(14.86 \%)$ & 141 & $36(25.53 \%)$ \\
\hline
\end{tabular}

\section{Discussion}

Surveys of the prevalence and antibacterial susceptibility patterns of bacterial isolates are important for determining appropriate empirical therapy for infections in critically-ill patients. Also, epidemiological analysis of patient data can be informative for appropriate management of patients in ICUs.

In our study most common site of infection is respiratory tract. Culture of 159 sample of tracheal aspirate was done in 2010 to 2012 . Among them
121 culture was positive and most frequent organism Acinetobacter was isolated in maximum $55(45.45 \%)$ samples followed by Psedomonas in 39 $(32.23 \%)$ samples, Proteus in $11(9 \%)$ samples, Klebsiella in 10(8.26\%) samples, E.Coli in $3(2.47 \%)$ samples and Streptococcus in 1(0.82\%) sample. A very high rate $27(22.31 \%)$ of resistant organism was identified, sensitive to only one antibiotic was $35(28.92 \%)$ and sensitive to many antibiotic was $59(48.76 \%)$. Among the antibiotic collistin (polymyxin E) was only sensitive for $23.14 \%$ 
organism, carbapenem $2.47 \%$ and tazobactampiperacillin combination $2.47 \%$.

In blood culture, among 148 samples 22(14.86\%) samples were positive. Among identified organism pseudomonas was $14(63.63 \%)$, acenatobacter $5(22.72 \%)$, E. coli 1(4.54\%) and salmonella 1(4.54\%). In urine culture, among 141 samples- 36(25.53\%) samples were positive. Among identified organism enterococcus was $8(22.22 \%)$, acenatobacter 7(19.44\%),Candida 6(16.66\%), E.coli 5(13.88\%), klebsiella 5(13.88\%) and pseudomonas 4(11.11\%).

In Asian countries including Indonesia, the most frequent pathogen isolated from infections in the ICU are P. aeruginosa, Klebsiella spp., E. coli, Enterococcus, and Staphylococcus aureus. For example, in 12 ICUs in seven Indian cities, overall $87.5 \%$ of all Staphylococcus aureus health care associated infections were caused by methicillinresistant strains, $71.4 \%$ of Enterobacteriaceae were resistant to ceftriaxone and $26.1 \%$ to piperacillintazobactam; $28.6 \%$ of the P. aeruginosa strains were resistant to ciprofloxacin, $64.9 \%$ to ceftazidime and $42.0 \%$ to imipenem ${ }^{19}$.

In Thailand the predominance causative pathogens in ICU, were the imipenem resistant P. aeruginosa, ceftazidime-resistant Acinetobacter baumannii, third-generation-cephalosporinresistant K. pneumoniae, and quinolone-resistant E. coli ${ }^{20}$. Another study performed at ICU of a tertiary care center in Saudi Arabia showed that the most frequent pathogens are Acinetobacter baumannii, P. aeruginosa, E. coli, K. pnemoniae ${ }^{21}$. Recently, similar studies were conducted in hospitals and several ICUs in Asian countries including Philippine ${ }^{22}$, India ${ }^{23,24,25,26} \operatorname{Iran}^{27,28}$, China $^{29}$, Malaysia ${ }^{30}$, Singapore ${ }^{31}$, and $\mathrm{Nepal}^{32}$, demonstrated that the most frequent microorganism derived from ICU samples were P. aeruginosa, Klebsiella spp. and Staphylococcus aureus. In Canada, the Canadian National Intensive Care Unit study conducted during 20052006, showed that P. aeruginosa, Staphylococcus aureus, Haemophilus influenzae, Enterococcus spp., Staphylococcus pneumoniae, and K. pneumoniae are the most common isolates recovered from clinical specimens in Canadian ICUs. Moreover, P. aeruginosa is the most frequent multi drug-resistant phenotype, which is resistance to three or more of the antibiotics including cefepime, piperacillin-tazobactam, meropenem, amikacin or gentamicin, and ciprofloxacin $^{33}$.

In our study showed that the most common pathogens identified from clinical specimen were Acinetobacter baumannii, P. aeruginosa, E. coli, Klebsiella pnemoniae and acenetobacter is the most frequent multidrug resistant phenotype and about $22.31 \%$ organisms were resistant to all available antibiotics in market. Ultimate fate of these patients is no need to mentioned. It's a grave situation. As we are being unable to treat many of these infections, so due important is to be given on prevention of infection and prevention of development antimicrobial resistance. Most sensitive antibiotic is polymixin- colistin. About one fourth organisms were resistant to carbanem which is also an alarming sign for us.

The prescribing of antibiotics in the ICU is usually empiric. Therefore, the ongoing surveillance of antibiotic susceptibility patterns of predominant bacteria is a fundamental effort to monitor changes in susceptibility patterns and to guide the clinician in choosing empirical or directed therapy appropriately, especially in ICU setting. Appropriate antibiotic utilization in ICU is crucial not only in ensuring an optimal outcome, but also in preventing the emergence of multi drug resistance bacteria. Therefore developing nation wide antibiotic policy and guidelines is essential to limit multidrug resistance and to maintain low level of resistance to newer antibiotics. Several societies have published guidelines for optimizing antibiotic use and curtailing antibiotic resistance in hospitals. Key components of these guidelines include multidisciplinary coordination between hospital administrators, clinicians, infectious disease specialists, infection control teams, microbiologist and hospital pharmacists.

In order to prevent the emergence and spread of antimicrobial resistance pathogens in ICU, the pattern of antimicrobial use has to be determined. A multidisciplinary approach is required to succeed in combating the problem. Hospital should monitor antimicrobial use to determine whether in ICUs or the entire hospital is overusing antimicrobials.

Introduction of strict and mandatory infection control program in hospital setting like proper hand washing before and after contact with 
patients, appropriate isolation of patients, standard guideline for disinfection and sterilization of medical equipment should be introduced.

This study conclude that most common site of infection was respiratory tract. Most prevailing organism was acinetobacter \& pseudomonas. Around one fourth organisms were resistant to all antibiotics. Around one fourth organisms are sensitive to only one antibiotic. Most sensitive antibiotic is polymixin- colistin. Around half of the organisms are sensitive to many antibiotics. Positive blood culture reports are lowest in comparison to others.

\section{References}

1. Marwick C, Davey P: Care bundles: the holy grail of infectious risk management in hospital? Curr Opin Infect Dis 2009; 22:364369

2. Hanberger H, Garcia-Rodriguez JA, Gobernado M, Goossens H, Nilsson LE, Struelens MJ: Antibiotic susceptibility among aerobic gram-negative bacilli in intensive care units in 5 European countries. French and Portuguese ICU Study Groups. JAMA 1999; 281:67-71

3. Vincent JL, Bihari DJ, Suter PM, Bruining HA, White J, Nicolas-Chanoin MH, Wolff M, Spencer RC, Hemmer M: The prevalence of nosocomial infection in intensive care units in Europe. Results of the European Prevalence of Infection in Intensive Care (EPIC) Study. EPIC International Advisory Committee. JAMA 1995; 274:639-644

4. Vandijck DM, Depaemelaere M, Labeau SO, Depuydt PO, Annemans L, Buyle FM, Oeyen S, Colpaert KE, Peleman RP, Blot SI, Decruyenaere JM: Daily cost of antimicrobial therapy in patients with Intensive Care Unitacquired, laboratory-confirmed bloodstream infection. Int J Antimicrob Agents 2008; 31:161-165

5. Blot S: Limiting the attributable mortality of nosocomial infection and multidrug resistance in intensive care units. Clin Microbiol Infect 2008; $14: 5-13$

6. Blot S, Depuydt P, Vandewoude K, De Bacquer D: Measuring the impact of multidrug resistance in nosocomial infection. Curr Opin Infect Dis 2007; 20: 391-396

7. Salgado CD, O'Grady N, Farr BM: Prevention and control of antimicrobialresistant infections in intensive care patients. Crit Care Med 2005; 33: 2373-2382

8. Cosgrove SE: The relationship between antimicrobial resistance and patient outcomes: mortality, length of hospital stay, and health care costs. Clin Infect Dis 2006, 42(Suppl 2):S82-S89

9. Figueiredo Costa S: Impact of antimicrobial resistance on the treatment and outcome of patients with sepsis. Shock 2008; 30: 23-29

10. Levy S.B. Factors impacting on the problems of antibiotic resistance. J. Antimicrob Chemotherapy, 20002; 49: 25- 30

11. Livermore, D.M. Minimising antibiotic resistance, Lancet Infect. Dis 2005; 5:450-459

12. Bacon, DJ.; Alm, RA.; Burr, DH.; Hu, L.; Kopecko, DJ.; Ewing, CP.; Trust, TJ. and Guerry. Involvement of a plasmid in virulence of Campylobacter jejuni 81-176. Infect Immun. Aug., 2000; 68(8):4384-90

13. Sakharkar, MK.; Jayaraman P, Soe WM, Chow VT, Sing LC, Sakharkar KR: In vitro combinations of antibiotics and phytochemicals against Pseudomonas aeruginosa. J MicrobiolImmunol Infect. Oct.2009; 42(5):364-70

14. Streit, JM.; Jones, RN.; Sader, HS. And Fritsche TR. Assessment of pathogen occurrences and resistance profiles among infected patients in the intensive care unit: report from the SENTRY Antimicrobial Surveillance Program (North America, 2001). Int J. Antimicrob Agents. Aug., 2004; 24(2):111-8

15. Nienke van de Sande-Bruinsma, Hajo Grundmann, Didier Verloo, EdineTiemersma, Jos Monen, Herman Goossens, MatusFerech, and the European Antimicrobial Resistance Surveillance System and Surveillance of Antimicrobial Consumption Project Groups. Antimicrobial Drug Use and Resistance in Europe. Emerg Infect Dis. November; 2008; 14(11): $1722-1730$ 
16. Kent Peters, N.; Dennis M. Dixon; Steven M. Holland and Anthony S. Fauci. The Research Agenda of the National Institute of Allergy and Infectious Diseases for Antimicrobial Resistance. The Journal of Infectious Diseases 2008; 197:1087-1093

17. Angulo, F.J.; Nunnery J.A. and Bair, H.D.. Antimicrobial resistance in zoonotic enteric pathogens. Rev. Sci. Technol. 2004; 2: 485-96

18. Sameera, M.; Al Johani ; Javed Akhter; Hanan Balkhy; Ayman El-Saed; Mousaad Younan and Ziad Memish (2010): Prevalence of antimicrobial resistance amonggram-negative isolates in an adult intensive care unit at a tertiary care center in Saudi Arabia. Ann Saudi Med 2010; 30(5): 364-369

19. Mehta A, Rosenthal VD, Mehta Y, Chakravarthy M, Todi SK, Sen N, et al. Device- associated nosocomial infection rates in intensive care units of seven Indian cities: findings of the International Nosocomial Infection Control Consortium (INICC). J Hosp Infect 2007; 67: 168-174

20. Thongpiyapoom S, Narong MN, Suwalak N, Jamulitrat S, Intaraksa P, Boonrat J, et al. Device associated infections and patterns of antimicrobial resistance in a medical-surgical intensive care unit in a university hospital in Thailand. J Med Assoc Thai 2004; 87: 819-824

21. Al Johani SM, Akhter J, Balkhy H, El-Saed A, Younan M, Memish Z. Prevalence of antimicrobial resistance among gramnegative isolates in an adult intensive care unit at a tertiary care center in Saudi Arabia. Ann Saudi Med 2010; 30: 364-369

22. Litzow JM, Gill CJ, Mantaring JB, Fox MP, MacLeod WB, Mendoza M, et al. High frequency of multidrug-resistant gramnegative rods in 2 neonatal intensive care units in the Philippines. Infect Control Hosp Epidemiol 2009; 30(6): 543-549

23. Shalini S, Kranthi K, Gopalkrishna Bhat K. Microbiological profile of nosocomial infection in the intensive care unit. J Clin Diagnostic Res 2010; 4(5): 3109-3012

24. Kaul S, Brahmadathan KN, Jagannati M, Sudarsanam TD, Pitchamuthu K, Abraham
OC, et al. One year trends in the gram-negative bacterial antibiotic susceptibility patterns in a medical intensive care unit in South India. Indian J Med Microbiol 2007; 25: 230-235

25. Tsering DC, Das S, Adhiakari L, Pal R, Singh TS. Extended spectrum beta-lactamase detection in gram-negative bacilli of nosocomial origin. J Glob Infect Dis 2009; 1: 87-92

26. Kumari HB, Nagarathna S, Chandramuki A. Antimicrobial resistance pattern among aerobic gram-negative bacilli of lower respiretory tract specimens of intensive care unit patients in a neurocentre. Indian J Chest Dis Allied Sci 2007; 49: 19-22

27. Jamshidi M, Javadpour S, Eftekhari TE, Moradi N, Jomehpour F. Antimicrobial resistance pattern among intensive care unit patients. Afr J Microbiol Res 2009; 3(10): 590594

28. Hassandeh P, Motamedifar M, Hadi N. Prevalent bacterial infection in intensive care units of Shiraz University of Medical Science Teaching Hospital, Shiraz Iran. Jpn J Infect Dis 2009; 62: 249-253

29. Cheng B, Xie G, Yao S, Wu X, Guo Q, Gu M, et al. Epidemiology of severe sepsis in critically ill surgical patients in ten university hospitals in China. Crit Care Med 2007; 35(11): 2538-2546

30. Gillani W, Sulaiman A, Nejad FB. Antibiotic resistance and therapeutic management of sepsis in a Malaysian Public Hospital. Australas Med J 2009; 1(14): 244-245

31. Hsu LY, Tan TY, Jureen R, Koh TH, Krishnan P, Lin RTP, et al. Antimicrobial drug resistance in Singapore hospitals. Emerg Infect Dis 2007; 13(12): 1944-1947

32. Shankar PR, Partha P, Dubey AK, Mishra P, Deshpande VY. Intensive care unit drug utilization in a teaching hospital in Nepal. Kathmandu Univ Med J 2005; 3(2): 130-137

33. Zanel GG, DeCorby M, Laing N, Weshnoweski B, Vashisht R, Tailor F, et al. Antimicrobialresistant pathogens in intensive care units in Canada: results of the Canadian National Intensive Care Unit (CAN-ICU) study, 20052006. Antimicrob Agents Chemother 2008; 52(4): 1430-1437 